\title{
Error Probability of BICM in Fading Channels: Uniform Interleaving Analysis
}

\author{
Alfonso Martinez \\ Centrum voor Wiskunde en Informatica \\ Postbus 94079, 1090 GB Amsterdam \\ The Netherlands \\ alfonso.martinezlieee.org
}

\author{
Albert Guillén i Fàbregas \\ Engineering Department \\ University of Cambridge \\ Cambridge, CB2 1PZ, UK \\ guillendieee.org
}

\begin{abstract}
This paper studies the average error probability of bit-interleaved coded modulation with uniform interleaving in fully-interleaved fading channels. At large signal-to-noise ratio, the dominant pairwise error events are mapped into symbols with Hamming weight larger than one, causing a flattening of the error probability. Closed-form expressions for the error probability with general modulations are provided. For interleavers of practical length, the flattening is noticeable only at very low values of the error probability.
\end{abstract}

\section{MOTIVATION AND SUMmary}

Whilst QPSK is equivalent to two parallel independent BPSK channels in the Gaussian channel, the equivalence fails in fading channels because of the statistical dependence between the quadrature components introduced by the fading coefficients. This dependence ensures that the error probability is dominated by the number of QPSK symbols with Hamming weight two. The asymptotic slope of the error probability is reduced and an error floor results, a phenomenon similar in nature to the floor appearing in turbo codes [1], [2].

More generally, bit-interleaved coded modulation (BICM) [3], [4], [5] is affected by the same phenomenon. For large signal-to-noise ratio (SNR), the error probability is determined by error events with a high diversity, so that the minimum Hamming weight of the code is distributed across the largest possible number of modulation symbols. Although "worse" error events with smaller diversity are present, they are weighted by a low error probability, and thus remain hidden for most practical purposes. We analyze this behaviour by studying the union bound to the error probability for general constellations over fully-interleaved fading. The saddlepoint approximation allows us to derive closed-form expressions for the pairwise error probability, which highlight the aforementioned floor effect. Finally, we estimate of the threshold SNR at which the error probability changes slope.

\section{Channel Model}

At the transmitter, a linear binary code of rate $R_{c}$ is used to generate codewords $\boldsymbol{c}=\left(c_{1}, c_{2}, \ldots, c_{\ell}\right)$ of length $\ell$, which are interleaved before modulation. Then, consecutive groups of $m$ interleaved bits $\left(c_{m(k-1)+1}, \ldots, c_{m k}\right)$, for $k=1, \ldots, n$, are

\footnotetext{
${ }^{1}$ This work was supported by the International Incoming Short Visits Scheme 2007/R2 of the Royal Society
}

mapped onto a modulation symbol $x_{k}$ using some mapping rule, such as binary reflected Gray labeling. We denote the modulation signal set by $\mathcal{X}$, its cardinality by $|\mathcal{X}|$, and the number of bits per symbol $m=\log _{2}|\mathcal{X}|$. The signal set is normalized to unit average energy. We also assume that $n \triangleq$ $\frac{\ell}{m}$ is an integer. We denote the inverse mapping function for labeling position $j$ as $c_{j}: \mathcal{X} \rightarrow\{0,1\}$, namely $c_{j}(x)$ is the $j$ th bit of symbol $x$. The sets $\mathcal{X}_{c_{1}, \ldots, c_{v}}^{j_{1}, \ldots, j_{v}}$ contain the symbols with bit labels in positions $j_{1}, \ldots, j_{v}$ equal to $c_{1}, \ldots, c_{v}$. For each input symbol the corresponding channel output $y_{k}$ is given by

$$
y_{k}=h_{k} \sqrt{\mathrm{SNR}} x_{k}+z_{k}, \quad k=1, \ldots, n
$$

where SNR is the average received signal-to-noise ratio, $z_{k}$ are independent samples of circularly-symmetric Gaussian noise of variance 1 , and $h_{k}$ are i. i. d. fading coefficients. We assume that the fading coefficient is known at the receiver, which implies that the phase of $h_{k}$ is irrelevant thanks to the circular symmetry of the noise. We also assume that the fading coefficients $h_{k}$ are drawn from a Nakagami distribution of parameter $m_{f}>0$ [6]. This distribution encompasses Rayleigh and AWGN channels, and approximates Rician fading.

\section{UNION BOUND FOR BICM}

At the receiver, we consider the maximum-metric BICM decoder which selects the codeword with largest metric $\prod_{k=1}^{n} q\left(x_{k}, y_{k}\right)$ [4]. The real-valued metric function $q\left(x_{k}, y_{k}\right)$ is computed by the demodulator for each symbol according to the formula

$$
q\left(x_{k}, y_{k}\right)=\prod_{j=1}^{m} q_{j}\left(c_{j}\left(x_{k}\right), y_{k}\right),
$$

where the bit metric $q_{j}\left(c_{j}(x)=c, y\right)$ is given by

$$
q_{j}\left(c_{j}(x)=c, y\right)=\sum_{x^{\prime} \in \mathcal{X}_{c}^{j}} p\left(y \mid x^{\prime}, h\right),
$$

where $p(y \mid x, h)=\frac{1}{\pi} e^{-|y-h \sqrt{\operatorname{SNR}} x|^{2}}$ is the channel transition probability density.

The word error rate, denoted by $P_{e}$, is the probability of selecting at the decoder a codeword different from the transmitted one. Similarly, the bit error rate $P_{b}$ is the average number of input bits in error out of the possible $\ell R_{c}$ 
corresponding to a codeword of length $\ell$. Exact expressions for $P_{e}$ or $P_{b}$ are difficult to obtain and one often resorts to bounding, such as the union bound [6]. In the union bound, the probability of an error event is bounded by the sum of the probabilities of all possible pairwise error events, where a codeword $\boldsymbol{c}^{\prime}$ other than the transmitted $\boldsymbol{c}$ has a larger metric. We define the pairwise score as

$$
\xi_{\mathrm{pw}} \triangleq \sum_{k=1}^{n} \sum_{j=1}^{m} \log \frac{q_{j}\left(c_{m(k-1)+j}^{\prime}, y_{k}\right)}{q_{j}\left(c_{m(k-1)+j}, y_{k}\right)} .
$$

The pairwise error probability $\operatorname{PEP}\left(\boldsymbol{c}^{\prime}, \boldsymbol{c}\right)$ between a reference codeword $c$ and the competitor codeword $c^{\prime}$ is given by $\operatorname{PEP}\left(\boldsymbol{c}^{\prime}, \boldsymbol{c}\right)=\operatorname{Pr}\left\{\xi_{\mathrm{pw}}>0\right\}$. By construction, the pairwise score is given by the sum of $n$ symbol scores,

$$
\xi_{k}^{\mathrm{s}} \triangleq \sum_{j=1}^{m} \log \frac{q_{j}\left(c_{m(k-1)+j}^{\prime}, y_{k}\right)}{q_{j}\left(c_{m(k-1)+j}, y_{k}\right)} .
$$

If the codewords have Hamming distance $d$, at most $d$ symbol scores are non zero. Further, each symbol score is in turn given by the sum of $m$ bit scores

$$
\xi_{k, j}^{\mathrm{b}} \triangleq \log \frac{q_{j}\left(c_{m(k-1)+j}^{\prime}, y_{k}\right)}{q_{j}\left(c_{m(k-1)+j}, y_{k}\right)} .
$$

Clearly, we only need to consider the non-zero bit and symbol scores. These scores are random variables whose density function depends on all the random elements in the channel, as well as the transmitted bits, their position in the symbol and the bit pattern. In order to avoid this dependence, and as done by Yeh et al. [7], a uniform interleaver [2] is added between the binary code and the mapper, so that the pairwise error probability is averaged over all possible ways of placing the $d$ bits in the $n$ modulation symbols. We distinguish these alternative placements by counting the number of symbols with weight $v$, where $0 \leq v \leq w^{\star}$ and $w^{\star}=\min (m, d)$. Denoting the number of symbols of weight $v$ by $n_{v}$, the symbol pattern $\rho_{n}$ is given by $\rho_{n}=\left(n_{0}, \ldots, n_{w^{\star}}\right)$. We also have that $\sum_{v=0}^{w^{\star}} n_{v}=n$ and $d=\sum_{v=1}^{w^{\star}} v n_{v}$.

For finite-length interleaving, the conditional pairwise error probability $\operatorname{PEP}\left(d, \rho_{n}\right)$ varies for every possible pattern. As done by Yeh et al. [7], averaging over all possible ways of choosing $d$ locations in a codeword we have the following union bounds.

$$
\bar{P}_{e} \leq \sum_{d} A_{d} \sum_{\rho_{n}} P\left(\rho_{n}\right) \operatorname{PEP}\left(d, \rho_{n}\right),
$$

where $A_{d}$ is the number of binary codewords of Hamming weight $d$ and $P\left(\rho_{n}\right)$ is the probability of a particular pattern $\rho_{n}$. We obtain the union bound on the bit error probability by replacing $A_{d}$ by $A_{d}^{\prime}=\sum_{j} \frac{j}{R_{c} \ell} A_{j, d}$, with $A_{j, d}$ being the number of codewords of Hamming weight $d$ generated with an input message of weight $j$. If a more detailed code spectrum were known, namely $A_{d, \rho_{n}}$ and $A_{j, d, \rho_{n}}$, respectively denoting the number of codewords with Hamming weight $d$ mapped onto the pattern $\rho_{n}$ and the number of such codewords with input weight $j$, similar expressions to those in Eq. (7) could be written without the averaging operation. A counting argument [7] gives the probability of the pattern $\rho_{n}, P\left(\rho_{n}\right) \triangleq \operatorname{Pr}\left(\rho_{n}=\right.$ $\left.\left(n_{0}, \ldots, n_{w^{\star}}\right)\right)$, as

$$
P\left(\rho_{n}\right)=\frac{\left(\begin{array}{c}
m \\
1
\end{array}\right)^{n_{1}}\left(\begin{array}{c}
m \\
2
\end{array}\right)^{n_{2}} \cdots\left(\begin{array}{c}
m \\
w^{\star}
\end{array}\right)^{n_{w^{\star}}}}{\left(\begin{array}{c}
m n \\
d
\end{array}\right)} \frac{n !}{n_{0} ! n_{1} ! n_{2} ! \ldots n_{w^{\star}} !}
$$

We remove the dependence of the pairwise error probability on the specific choice of modulation symbols by averaging over all possible choices. This method consists of adding to every transmitted codeword $\boldsymbol{c} \in \mathcal{C}$ a random binary word $\boldsymbol{d} \in$ $\{0,1\}^{n}$ known by the receiver. This is equivalent to scrambling the output of the encoder by a sequence known at the receiver. Scrambling guarantees the symbols corresponding to two $m$ bit sequences $\left(c_{1}, \ldots, c_{m}\right)$ and $\left(c_{1}^{\prime}, \ldots, c_{m}^{\prime}\right)$ are mapped to all possible pairs of modulation symbols differing in a given Hamming weight, hence making the channel symmetric. In [4], [7], the scrambler role was played by a random choice between a mapping rule $\mu$ and its complement $\bar{\mu}$ with probability $1 / 2$ at every channel use. Scrambling is the natural extension of this random choice to weights larger than 1 .

The cumulant transform [8] is an equivalent representation of the probability distribution of a random variable; the distribution can be recovered by an inverse Fourier transform. Consider a non-zero symbol score $\Xi^{\mathrm{s}}$ of Hamming weight $1 \leq v \leq m$, i.e., the Hamming weight between the binary labels of the reference and competitor symbols is $v$. The cumulant transform of $\Xi^{\mathrm{s}}$ is $\kappa_{v}(s) \triangleq \log \mathrm{E}\left[e^{s \Xi^{\mathrm{s}}}\right]$, and more explicitly given by

$$
\kappa_{v}(s)=\log \left(\frac{1}{\left(\begin{array}{c}
m \\
v
\end{array}\right)} \sum_{j} \frac{1}{2^{v}} \sum_{c \in\{0,1\}^{v}} \mathrm{E}\left[\frac{\prod_{i=1}^{v} q_{j_{i}}\left(\bar{c}_{j_{i}}, Y\right)^{s}}{\prod_{i=1}^{v} q_{j_{i}}\left(c_{j_{i}}, Y\right)^{s}}\right]\right),
$$

where $\boldsymbol{j}=\left(j_{1}, \ldots, j_{v}\right)$ is a sequence of $v$ bit indices, the bit $\bar{c}$ is the binary complement of $c$, and $y$ are the channel outputs with bit $v$-tuple $c$ transmitted at positions in $\boldsymbol{j}$. The cumulant transform of the pairwise score $\Xi_{\mathrm{pw}}\left(\rho_{n}\right)$ is given by

$$
\kappa_{\mathrm{pw}}\left(s, \rho_{n}\right) \triangleq \log \mathrm{E}\left[e^{s \Xi_{\mathrm{pw}}\left(\rho_{n}\right)}\right]=\sum_{v=1}^{w^{\star}} n_{v} \kappa_{v}(s) .
$$

The expectation in (9) is done according to $p_{\boldsymbol{j}}(y \mid \boldsymbol{c})=$ $\frac{1}{2^{m-v}} \sum_{x \in \mathcal{X}_{c_{1}, \ldots, c_{v}}^{j_{1}, \ldots, j_{v}}} p(y \mid x, h)$.

An important feature of the cumulant transform is that the tail probability is to great extent determined by the cumulant transform around the saddlepoint $\hat{s}$, defined as the value of $s$ for which $\kappa^{\prime}(\hat{s}) \triangleq \frac{d \kappa(s)}{d s}=0$. Indeed, in our notation the Chernoff bound is given by $\operatorname{PEP}\left(d, \rho_{n}\right) \leq e^{\kappa_{\mathrm{pw}}\left(\hat{s}, \rho_{n}\right)}$. Then the saddlepoint approximation to $\operatorname{PEP}\left(d, \rho_{n}\right)$ is given by

$$
\operatorname{PEP}\left(d, \rho_{n}\right) \simeq \frac{1}{\hat{s} \sqrt{2 \pi \kappa_{\mathrm{pw}}^{\prime \prime}\left(\hat{s}, \rho_{n}\right)}} e^{\kappa_{\mathrm{pw}}\left(\hat{s}, \rho_{n}\right)},
$$

where the saddlepoint $\hat{s}$ is the root of the equation $\kappa_{\mathrm{pw}}^{\prime}\left(s, \rho_{n}\right)=0$. 
A particularly important case arises when $v=1$, i.e., when the binary labels of the reference and competitor symbols in the symbol pairwise score differ only by a single bit and all $d$ different bits are mapped onto different constellation symbols. This is the case for interleavers of practical length. As noticed in [4], [9], this simplifies significantly the analysis. The cumulant transform of the symbol score with $v=1$, denoted by $\Xi_{1}^{\mathrm{b}}$, is given by

$$
\kappa_{1}(s)=\log \left(\frac{1}{m} \sum_{j=1}^{m} \frac{1}{2} \sum_{c \in\{0,1\}} \mathrm{E}\left[\frac{q_{j}(\bar{c}, Y)^{s}}{q_{j}(c, Y)^{s}}\right] \cdot\right),
$$

We denote the corresponding pairwise error probability by $\mathrm{PEP}_{1}(d)$. As noticed in [9], This expression is related, but not identical to, the ones appearing in [4], [7]. These authors consider an approximation to the bit decoding metric $q_{j}(c, y)$ whereby only one of the summands in Eq. (3) is kept. Even though this approximation slightly simplifies the analysis, it leads to large inaccuracies for mappings other than Gray mapping. In this paper, we consider the full bit metric.

As shown in Appendix I, the probability that all bit scores are independent approaches 1 as $1-\frac{d(d-1)}{2 \ell}(m-1)$ for large interleaver lengths. In general, the effect of the dependence across the bits belonging to the same symbol must be taken into account.

\section{LARGE-SNR ANALYSIS}

In this section, we present a large-SNR analysis of the union bound on the error probability with general constellations and mappings, and estimate the signal-to-noise ratio at which the slope of the error probability changes. Since we are interested in large values of SNR, we shall be working with an asymptotic approximation to the error probability. In particular, our analysis is based on an extension of the results in [4], [9] for the asymptotic behaviour of the error probability in the Rayleigh-fading channel. We will provide such asymptotic expressions for the symbols scores of varying weight and determine the value of SNR at which the various approximations to the error probability cross.

In [4], Caire et al. considered the Rayleigh fading at large SNR, and derived the following approximation to the cumulant transform of the bit score,

$$
\kappa_{1}(s) \simeq-\log \left(\frac{d_{\mathrm{h}}^{2}}{4} \mathrm{SNR}\right),
$$

where $d_{\mathrm{h}}^{2}$ is a harmonic distance given by

$$
d_{\mathrm{h}}^{2}=\left(\frac{1}{m 2^{m}} \sum_{c=0}^{1} \sum_{j=1}^{m} \sum_{x \in \mathcal{X}_{c}^{j}} \frac{1}{\left|x-x^{\prime}\right|^{2}}\right)^{-1},
$$

where $x^{\prime}$ is the closest symbol in the constellation $\mathcal{X}_{\bar{c}}^{j}$ to $x$. Eq. (13) may be used in the Chernoff bound to give an approximation to the error probability at large SNR. It was found in [4] that this gives a good approximation. Using that $\hat{s}=\frac{1}{2}$, that $\lim _{\mathrm{SNR} \rightarrow \infty} \kappa_{1}(\hat{s})=8 m_{f}$ [9] and the saddlepoint approximation in Eq. (11), we obtain the large SNR heuristic approximation

$$
\operatorname{PEP}_{1}(d) \simeq \frac{1}{2 \sqrt{\pi d}}\left(\frac{4}{d_{\mathrm{h}}^{2} \mathrm{SNR}}\right)^{d} .
$$

In Appendix II we extend the analysis in [4] to the cumulant transform of the BICM symbol score of weight $v$ for Nakagami- $m_{f}$ fading, and obtain the following limit

$$
\kappa_{v}(\hat{s}) \simeq-m_{f} \log \left(\frac{d_{\mathrm{h}}^{2}(v)}{4 m_{f}} \mathrm{SNR}\right),
$$

where $d_{\mathrm{h}}^{2}(v)$ is a generalization of the harmonic distance given by

$$
d_{\mathrm{h}}^{2}(v)=\left(\frac{1}{\left(\begin{array}{c}
m \\
v
\end{array}\right) 2^{m}} \sum_{c} \sum_{j} \sum_{x \in \mathcal{X}_{j}^{c}}\left(\frac{\left|\sum_{i=1}^{v}\left(x-x_{i}^{\prime}\right)\right|}{\sum_{i=1}^{v}\left|x-x_{i}^{\prime}\right|^{2}}\right)^{2 m_{f}}\right)^{-\frac{1}{m_{f}}} .
$$

For a given $x, x_{i}^{\prime}$ is the $i$-th symbol in the sequence of $v$ symbols $\left(x_{1}^{\prime}, \ldots, x_{v}^{\prime}\right)$ which have binary label $\bar{c}_{j_{i}}$ at position $j_{i}$ and for which the ratio $\frac{\left|\sum_{i=1}^{v}\left(x-x_{i}^{\prime}\right)\right|}{\sum_{i=1}^{v}\left|x-x_{i}^{\prime}\right|^{2}}$ is minimum among all possible such sequences. For $m_{f}=1$ and $v=1$ we recover the harmonic distance $d_{\mathrm{h}}^{2}$ above.

As it happened with the bit score and $\operatorname{PEP}_{1}(d)$, Eq. (16) may be used in the Chernoff bound or in the saddlepoint approximation in Eq. (11), to obtain a heuristic approximation to the pairwise error probability for large SNR, namely

$$
\operatorname{PEP}_{\mathrm{H}}\left(d, \rho_{n}\right) \simeq \frac{1}{2 \sqrt{\pi m_{f} \sum_{v \geq 1} n_{v}}} \prod_{v=1}^{m}\left(\frac{4 m_{f}}{d_{\mathrm{h}}^{2}(v)} \frac{1}{\mathrm{SNR}}\right)^{n_{v} m_{f}} .
$$

For the sake of simplicity, we disregard the effect of the coefficient $\frac{1}{\hat{s} \sqrt{2 \pi \kappa_{\mathrm{pw}}(\hat{s})}}$ in our analysis of the threshold SNR.

For sufficiently large signal-to-noise ratio, the error probability is determined by the worst possible distribution pattern $\rho_{n}$ of the $d$ bits onto the $n$ symbols, that with the largest tail probability for the pairwise score. Then, since $d=$ $\sum_{v=1}^{w^{*}} n_{v} v$ by construction, we can view the pattern $\rho_{n}=$ $\left(n_{0}, n_{1}, \ldots, n_{w^{\star}}\right)$ as a (non-unique) representation of the integer $d$ as a weighted sum of the integers $\left\{0,1,2, \ldots, w^{\star}\right\}$. By construction, the sum $\sum_{v \geq 1} n_{v}$ is the number of nonzero Hamming weight symbols in the candidate codeword. Clearly, the lowest $\sum_{v \geq 1} n_{v}$ gives the worst (flattest) pairwise error probability in the presence of fading. By equating the contribution of the steepest and flattest terms, we obtain an equation for $\mathrm{SNR}_{\mathrm{th}}$,

$$
\begin{aligned}
& \left(P\left(\rho_{0}\right)\left(\frac{4 m_{f}}{d_{\mathrm{h}}^{2}(1)} \frac{1}{\mathrm{SNR}_{\mathrm{th}}}\right)^{m_{f}}\right)^{d} \\
& =\frac{1}{\mathrm{SNR}_{\mathrm{th}}^{m_{f} \sum_{v=1}^{m} n_{v}}} \sum_{\rho_{n}: \min \sum_{v} n_{v}} P\left(\rho_{n}\right) \prod_{v=1}^{m}\left(\frac{4 m_{f}}{d_{\mathrm{h}}^{2}(v)}\right)^{n_{v} m_{f}} .
\end{aligned}
$$


The left-hand side corresponds to the steepest pairwise error probability, namely $\operatorname{PEP}_{1}(d)$, weighted by the probability that all bit scores are independent, denoted by $P\left(\rho_{0}\right)$. The righthand side corresponds to the largest pairwise error probability with smallest number of non-zero symbol scores, that is among all possible patterns $\rho_{n}$ with minimum $\sum_{v} n_{v}$. Note that the exponent of SNR is $-m_{f} \sum_{v} n_{v}$, and thus has the lowest possible diversity, as it should.

From Eq. (19), we can extract the value of $\mathrm{SNR}_{\text {th }}$ as

$\mathrm{SNR}_{\mathrm{th}} \simeq 4 m_{f}\left(\sum_{\rho_{n}: \min \sum_{v} n_{v}} \frac{P\left(\rho_{n}\right)\left(d_{\mathrm{h}}^{2}(1)\right)^{d}}{P\left(\rho_{0}\right) \prod_{v}\left(d_{\mathrm{h}}^{2}(v)\right)^{n_{v}}}\right)^{-\frac{1}{m_{f}\left(d-\sum_{v} n_{v}\right)}}$

As we observe in Figure 1, there is a slope change for 8PSK with Rayleigh fading and Gray labeling; the code is the optimum 8-state rate-2/3 convolutional code, with $d_{\min }=4$. This effect is due to the probability of having symbol scores of Hamming weight larger than 1 . The figure depicts simulation results (for interleaver sizes $\ell=90,3000$ ) together with the saddlepoint approximations for finite $\ell$ (for $\ell=90,300,3000$ ), infinite interleaving (with $\mathrm{PEP}_{1}(d)$ ), and with the heuristic approximation $\operatorname{PEP}_{\mathrm{H}}\left(d \rho_{n}\right)$ (only for $\ell=90$ and $d=4$ ). For 8-PSK with Gray mapping, evaluation of Eq. (17) gives $d_{\mathrm{h}}^{2}(1)=0.7664, d_{\mathrm{h}}^{2}(2)=1.7175$, and $d_{\mathrm{h}}^{2}(3)=2.4278$. Table I gives the values of $P\left(\rho_{n}\right)$ for the various patterns $\rho_{n}$.

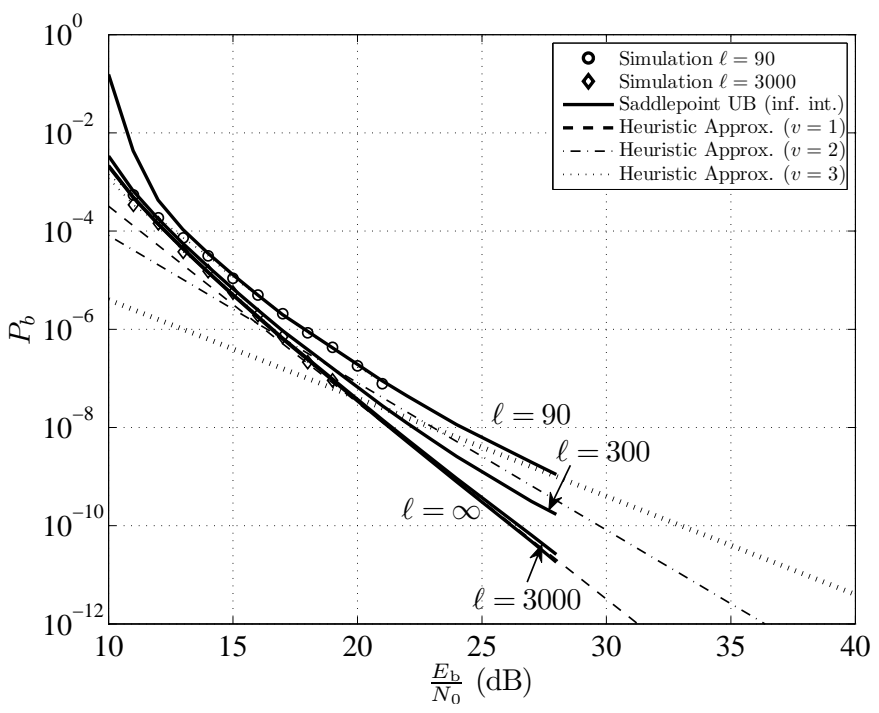

Fig. 1. Bit error probability union bounds and bit-error rate simulations of 8-PSK with the 8-state rate-2/3 convolutional code in a fully-interleaved Rayleigh fading channel. Interleaver length $\ell=90$ (circles) and $\ell=3000$ (diamonds). In solid lines, the saddlepoint approximation union bounds for $\ell=90, \ell=300, \ell=3000$ and for infinite interleaving, with $\operatorname{PEP}_{1}(d)$. In dashed, dashed-dotted, and dotted lines, the heuristic approximations with weight $v=1,2,3$ respectively.

Table I also gives the threshold $\mathrm{SNR}_{\text {th }}$ given in Eq. (20) for all possible values of $\sum_{v \geq 1} n_{v}$, not only for the worst case. We observe that the main flattening of the error probability takes place at high SNR. This effect essentially disappears for interleavers of practical length: for $\ell=300$ (resp. $\ell=3000$ ) the error probability at the first threshold is about $10^{-8}$ (resp. $10^{-12}$ ). The saddlepoint approximation is remarkably precise; the heuristic approximation $\operatorname{PEP}_{\mathrm{H}}\left(d_{\mathrm{dmin}}, \rho_{n}\right)$ also gives very good results.

TABLE I

ASYMPTOTIC ANALYSIS FOR 8-PSK WITH VARYING INTERLEAVER LENGTH $\ell=3 n$ AND MINIMUM DISTANCE $d=4$.

\begin{tabular}{lccc}
\hline Pattern $\rho_{n}$ & $\ell$ & $P\left(\rho_{n}\right)$ & Threshold $\frac{E_{\mathrm{b}}}{N_{0}}(\mathrm{~dB})$ \\
\hline$(n-4,4,0,0)$ & $\ell=90$ & 0.8688 & N/A \\
& $\ell=300$ & 0.9602 & N/A \\
& $\ell=3000$ & 0.9960 & N/A \\
\hline$(n-3,2,1,0)$ & $\ell=90$ & 0.1287 & 16.0 \\
& $\ell=300$ & 0.0396 & 21.5 \\
& $\ell=3000$ & 0.0040 & 31.6 \\
\hline$(n-2,0,2,0)$, & $\ell=90$ & 0.0015 & 20.5 \\
$(n-1,1,0,1)$ & $\ell=300$ & 0.0002 & 26.0 \\
& $\ell=3000$ & $2 \cdot 10^{-6}$ & 39.1 \\
\hline
\end{tabular}

\section{CONCLUSions}

We have studied the large-SNR behavior of the error probability of BICM over fully-interleaved fading channels. Our analysis reveals that the pairwise error probability is asymptotically dominated by the number pairwise error symbols with Hamming weight larger than one, yielding an error floor. We have derived closed-form approximations to this error probability, as well as for the SNR value at which the error floor appears. For practical code lengths, the error floor appears at very low error rates.

\section{APPENDIX I \\ Probability of All-One Sequence}

We use Stirling's approximation to the factorial, $n ! \simeq$ $n^{n} e^{-n} \sqrt{2 \pi n}$, to Eq. (8) to obtain

$$
\begin{aligned}
P_{\text {ind }} & \triangleq P\left(n_{1}=d, n_{2}=0, \ldots, n_{w^{\star}}=0\right) \\
& =\frac{\ell^{n}}{(\ell-m d)^{n-d+\frac{1}{2}}} \frac{(\ell-d)^{\ell-d+\frac{1}{2}}}{\ell^{\ell}},
\end{aligned}
$$

with the obvious simplifications and combinations. Extracting a factor $\ell$ in $(\ell-d)$ and $(\ell-m d)$, and cancelling common powers of $\ell$ in numerator and denominator, we get

$$
P_{\text {ind }} \simeq\left(1-\frac{d}{n}\right)^{-n+d-\frac{1}{2}}\left(1-\frac{d}{\ell}\right)^{\ell-d+\frac{1}{2}} .
$$

We now take logarithms, and use Taylor's expansion of the logarithm, $\log (1+t) \simeq t-\frac{1}{2} t^{2}$, in the right-hand side of Eq. (23). Discarding all powers of $\ell$ higher than $\ell^{-2}$, and combining common terms, we obtain

$$
\log P_{\text {ind }} \simeq-\frac{m d^{2}}{2 \ell}+\frac{d}{2 n}+\frac{d^{2}}{2 \ell}-\frac{d}{2 \ell}=-\frac{d(d-1)}{2 \ell}(m-1) .
$$

Finally, recovering the exponential, $P_{\text {ind }} \simeq e^{-\frac{d(d-1)}{2 \ell}(m-1)}$. 


\section{APPENDIX II \\ CUMULANT TRANSFORM Limits}

We wish to compute the limit $\ell_{v}(s) \triangleq \lim _{\mathrm{SNR} \rightarrow \infty} \frac{e^{\kappa_{v}(\hat{s})}}{\mathrm{SNR}^{-m_{f}}}$, given by

$$
\begin{aligned}
& \ell_{v}(s)= \\
& \lim _{\mathrm{SNR} \rightarrow \infty} \frac{1}{\mathrm{SNR}^{-m_{f}}}\left(\frac{1}{2^{v}\left(\begin{array}{c}
m \\
v
\end{array}\right)} \sum_{j, \boldsymbol{c}} \mathrm{E}\left[\frac{\prod_{i=1}^{v} q_{j_{i}}\left(\bar{c}_{j_{i}}, Y\right)^{s}}{\prod_{i=1}^{v} q_{j_{i}}\left(c_{j_{i}}, Y\right)^{s}}\right]\right) .
\end{aligned}
$$

We can rewrite the denominator as

$$
\begin{aligned}
\prod_{i=1}^{v} q_{j_{i}}\left(\bar{c}_{j_{i}}, y\right) & =\prod_{i=1}^{v}\left(\sum_{x^{\prime} \in \mathcal{X}_{\bar{c}}^{j_{i}}} e^{-\left|H \sqrt{\operatorname{SNR}}\left(X-x^{\prime}\right)+Z\right|^{2}}\right) \\
& =\sum_{\boldsymbol{x}^{\prime}} \prod_{i=1}^{v} e^{-\left|H \sqrt{\operatorname{SNR}}\left(X-x_{i}^{\prime}\right)+Z\right|^{2}}
\end{aligned}
$$

where $\boldsymbol{x}^{\prime}$ is one of all possible sequences of $v$ modulation symbols, with symbol at index $j_{i}$ drawn from the set $\mathcal{X}_{\bar{c}_{j_{i}}}^{j_{i}}$. A similar formula holds for the denominator, now with symbols drawn from the set $\mathcal{X}_{c_{j_{i}}}^{j_{i}}$. Expanding the exponent in Eq. (27), we obtain

$$
\begin{aligned}
& \prod_{i=1}^{v} q_{j_{i}}\left(\bar{c}_{j_{i}}, Y\right)= \\
& \sum_{\boldsymbol{x}^{\prime}} e^{-|H| \operatorname{SNR} \sum_{i=1}^{v}\left|X-x_{i}^{\prime}\right|^{2}+2 \sqrt{\operatorname{SNR}} \operatorname{Re}\left(\sum_{i=1}^{v} H\left(X-x_{i}^{\prime}\right) Z^{*}\right)+v|Z|^{2}} .
\end{aligned}
$$

As done in [4], [9], we keep only the dominant summand in the bit scores $q_{j_{i}}(\cdot, y)$ appearing in numerator and denominator. For a given $x$, this summand corresponds to the sequence $\boldsymbol{x}^{\prime}$ having the smallest possible value of the ratio $\frac{\left|\sum_{i=1}^{v}\left(x-x_{i}^{\prime}\right)\right|}{\sum_{i=1}^{v}\left|x-x_{i}^{\prime}\right|^{2}}$. In particular, in the denominator all the symbols in the sequence coincide with $x$. We now carry out the expectation over $Z$. Completing squares, and using that the formula for the density of Gaussian noise, we have that

$$
\begin{gathered}
\int \frac{1}{\pi} e^{-|z|^{2}} e^{-\sum_{i=1}^{v} s\left(\operatorname{SNR}|H|^{2}\left|X-X_{i}^{\prime}\right|^{2}+2 \sqrt{\operatorname{SNR}} \operatorname{Re}\left(H\left(X-X_{i}^{\prime}\right) z^{*}\right)\right)} d z \\
=e^{-\operatorname{SNR}|H|^{2}\left(s \sum_{i=1}^{v}\left|X-X_{i}^{\prime}\right|^{2}-s^{2}\left|\sum_{i=1}^{v}\left(X-X_{i}^{\prime}\right)\right|^{2}\right)} .
\end{gathered}
$$

In turn, the expectation over $h$ of this quantity yields [11]

$$
\left(1+\left(s \sum_{i=1}^{v}\left|X-X_{i}^{\prime}\right|^{2}-s^{2}\left|\sum_{i=1}^{v}\left(X-X_{i}^{\prime}\right)\right|^{2}\right) \frac{\mathrm{SNR}}{m_{f}}\right)^{-m_{f}} .
$$

We next turn back to the limit of large SNR. We have that

$\ell_{v}(s)=$

$\frac{m_{f}^{m_{f}}}{2^{m}\left(\begin{array}{c}m \\ v\end{array}\right)} \sum_{j, \boldsymbol{c}} \sum_{x \in \mathcal{X}_{\boldsymbol{c}}^{j}}\left(s \sum_{i=1}^{v}\left|x-x_{i}^{\prime}\right|^{2}-s^{2}\left|\sum_{i=1}^{v}\left(x-x_{i}^{\prime}\right)\right|^{2}\right)^{-m_{f}}$.

For each summand, the optimizing $s$ is readily computed to be $\hat{s}=\frac{\sum_{i=1}^{v}\left|x-x_{i}^{\prime}\right|^{2}}{2\left|\sum_{i=1}^{v}\left(x-x_{i}^{\prime}\right)\right|^{2}}$, which gives

$$
\ell_{v}(\hat{s})=\frac{1}{2^{m}\left(\begin{array}{c}
m \\
v
\end{array}\right)} \sum_{j, c} \sum_{x \in \mathcal{X}_{c}^{j}}\left(\frac{4 m_{f}\left|\sum_{i=1}^{v}\left(x-x_{i}^{\prime}\right)\right|^{2}}{\left(\sum_{i=1}^{v}\left|x-x_{i}^{\prime}\right|^{2}\right)^{2}}\right)^{m_{f}}
$$

\section{REFERENCES}

[1] C. Berrou, A. Glavieux, and P. Thitimajshima, "Near Shannon limit error-correcting coding and decoding: Turbo-codes." in IEEE Int. Conf. Commun., Geneva, vol. 2, 1993.

[2] S. Benedetto and G. Montorsi, "Unveiling turbo codes: some results on parallel concatenated coding schemes," IEEE Trans. Inf. Theory, vol. 42, no. 2, pp. 409-428, 1996.

[3] E. Zehavi, "8-PSK trellis codes for a Rayleigh channel," IEEE Trans. Commun., vol. 40, no. 5, pp. 873-884, 1992.

[4] G. Caire, G. Taricco, and E. Biglieri, "Bit-interleaved coded modulation," IEEE Trans. Inf. Theory, vol. 44, no. 3, pp. 927-946, 1998.

[5] A. Guillén i Fàbregas, A. Martinez and G. Caire, "Bit-Interleaved Coded Modulation," Foundations and Trends in Communications and Information Theory, Now Publishers, vol. 5, no. 1-2, pp 1-153, 2008. http:/dx.doi.org/10.1561/0100000019

[6] J. G. Proakis, "Digital Communications," McGraw-Hill USA, 4th edition, 2001.

[7] P. Yeh, S. Zummo, and W. Stark, "Error probability of bit-interleaved coded modulation in wireless environments," IEEE Transactions on Vehicular Technology, vol. 55, no. 2, pp. 722-728, 2006.

[8] R. W. Butler, Saddlepoint Approximations with Applications. Cambridge University Press, 2007.

[9] A. Martinez, A. Guillén i Fàbregas, and G. Caire, "Error probability analysis of bit-interleaved coded modulation," IEEE Trans. Inf. Theory, vol. 52, no. 1, pp. 262-271, Jan. 2006.

[10] S. Zummo, P. Yeh, and W. Stark, "A Union Bound on the Error Probability of Binary Codes Over Block Fading Channels," IEEE Transactions on Vehicular Technology, vol. 54, no. 6, pp. 2085 - 2093, 2005.

[11] A. Martinez, A. Guillén i Fàbregas, and G. Caire, "A closed-form approximation for the error probability of BPSK fading channels," IEEE Trans. Wireless Commun., vol. 6, no. 6, pp. 2051-2056, Jun. 2007. 